\title{
The Design of Distributed-Server-Technology-Based Network Communication Software
}

\author{
Shunting Su \\ 1.Northeast Normal University,2.Jilin Teachers Institute \\ of Engineering and Technology Changchun, China \\ Email:191537788@qq.com
}

\author{
Nianfeng Li, Yinghua Lv \\ Changchun University, Northeast Normal University \\ Changchun, China \\ Email28721961@qq.com, 38891970@qq.com
}

\begin{abstract}
Given that the centralized-servers-technology-based network communication software like QQ and MSN are high on cost and limited on users' control right, this thesis presents a designing scheme of the distributed-servers-technology-based network communication software. The design follows consistently the idea of distributed servers technology and makes further improvement, emphasizing the designing idea that client side is server. The running process and protocol idea was presented in this thesis, as well as the third-level designs that are based on the idea.
\end{abstract}

Keywords-Distributed servers, Network programming, Agreement

\section{THE ANALYSIS OF THE DESIGNING IDEA OF THE} EXISTING COMMUNICATION SOFTWARE

The major existing network communication software technology include: centralized server technology, pure P2P (peer-to-peer) technology and distributed servers technology. Among them, the pure P2P technology is sought after most by the technology group. However, although a lot of efforts have been plunged into it, it still has got no relatively perfect way to achieve free network communication due to the flaw of its designing structure and limited technology. Thus there has not been a good communication product coming into being so far. As for QQ and MSN which are based on centralized server technology, they are currently popular and familiar among users.

In the history of China's software, QQ is absolutely a miracle, which you can find in every home computer or those of Internet bars. It is such an essential software that the Internet bar owner even say that they in will face bankruptcy if there is no QQ in the computers. To get a knowledge of QQ, we must first learn something about OICQ, and ICQ unavoidably.

In the summer of 1996, three Israeli young men named Weisg, Vadi, and Goldfinger respectively got together to decide to develop a kind of software to achieve instant communication on the Internet which features instant communication. So there came the designing idea of ICQ (the pronunciation of which is similar to "I Seek You"), which was then used for their personal communication tool and was like their personal "plaything”. They set up a small company named Mirabilis, and provided ICQ service to registered users. Early in 1999, a rush of online instant communication software sprang up in China that imitated ICQ, such as the PICQ, OICQ, and OMMO. OICQ of Tencent company, out of other instant communication software, won many users on account of the proper design and simple user operation. To avoid legal dispute with ICQ, OICQ was changed by Tencent Company to the name of QQ in 2000.

Each type of centralized-server-technology-based network communication software employs the same designing idea, that is, make one or certain computers serve as a server on which the server side software is operated. When user A wants to communicate with other users with the means of this communication software, first ,he needs operate a client side software, through which he registers his personal information (basic information and IP address) and logging information (whether visible) into the online users database at the server side. Then the server side will feed the information of related users (friends, strangers, someone on the blacklist) of user A back to user A. Then it is a matter of data transmission between use A-side software, server software, and other users-side software as is stipulated in the previous protocol.

As is aforementioned, this type of centralized-serverbased communication software is costly and the owner of the server impose commercial spam to the software users.very carefully check a printed copy.

\section{ThE DESIGN OF THE DistribUTED-SERVER-}

TECHNOLOGY-BASED COMMUNICATION SOFTWARE

The distributed server technology is the most promising technology in the existing network structure. Almost all the popular P2P software employs this technology more or less such as EDONGKEY and JXTA. They are just different in names.

\section{A. Technical advantages}

The distributed server technology brings a lot of benefits to network communication software. Among them, the most evident and important ones are:

(1) The load of the whole system is distributed to servers around the world, so there is no need to worry about the high cost of the centralized server; 
(2) There is more freedom of users' control right because the server is not controlled by one company or one group, so there is no need to worry about the control of a certain company due to commercial factors;

(3) The application spectrum is larger than the pure P2P technology.

Certainly, the distributed technology is more difficult to achieve than the centralized server technology, but easier than pure P2P technology. Therefore, to achieve P2P technology, we need first consider the distributed server technology which is relatively proper in all aspects.

\section{B. Designing idea}

The distributed-server-technology-based network communication software follows consistently the idea of distributed serve, and is further improved, and presents the designing idea that user is server. Under such designing idea, all the user notes of the whole software system are at the same level.

The distributed-server-technology-based network communication software does not depend on any server and runs according to the Internet environment and users' order. Users can make a server by themselves and provide service for other user notes that cannot make a server. Users also can enjoy the service provided by other users, and at the same time, provide service within their power for those who cannot make a server.

Therefore, there is no special server or special client side in the whole running process of the software system, in which all the users use the same software. Based on this idea, the software employs the following running process.

Assume the software that can achieve the abovementioned function is called DSC. First, when user A begins to run DSC, it will send a broadcast message on the Internet to seek whether a server exists. If so (we make it s1), then user A, as a client side, can connect with server s1, and get access to the information of other users on the server s1, and then connect with other users; at the same time, user A can also, as server s2, provide service to other users when user A needs register its server s2 identity on the server s1. If there is no server running (which means other users have not begun operation), then user A needs act as temporary server s1 till he wants to exit from the program or wants to stop the service, and at this time, if server s2 exists, then s1 will send information of all the servers group to s2. If we can make a communication protocol by strictly following the abovementioned idea, then the whole DSC net can runs smoothly without depending on any centralized server or impacting the complete functions of network communication.

\section{Protocol makeup}

Based on the above-mentioned designing idea, we can get the protocol makeup of the distributed-server-technologybased network communication software system. The consummation of the design needs four types of protocols: UIDP (User Information Discovery Protocol), GRP (General Request Protocol), RP (Routing Protocol) and GCP (Group Control Protocol).
1) UIDP (User Information Discovery Protocol): UIDP is used to release users' feature information and get access to information of other users. It allows a user to find the multicast information (including ID, status and resources) of other users. IP Multicast is a one-to-many message transmission protocol, which is used to send the group address of the data copy. In the application program of $\mathrm{P} 2 \mathrm{P}$, IP Multicast has two merits. First, a peer can send messages without knowing the receiver's address because the multicast uses a group address rather than IP address. Therefore, all the peers in the multicast network could respond to the peer that sends messages, and send back the relative information of the search results and even their own IP addresses (these IP addresses are used to ask peer to connect directly). Second, IP Multicast reduces the use of bandwidth because all the peers can see a single message and they have no need to send a message copy to every peer. This is even more important when sending a mess of data to a group of peers. This type of protocol sometimes can be used as a ping order to explore the basic information and status of other users on the network. UIDP message is freetexted, which could be used to be extended to provide control. It employs GRP to send and broadcast more general requests.

2) GRP (General Request Protocol): GRP allows users to send more general requests and can receive response of the request; at the same time, it can distribute the requests to one or more matched users. GRP is a basic communication protocol which runs with a request/ reply format. To use this protocol, user needs provide a research target and a request message that can be understood by the target user. GRP is used to support other protocols (UIDP and GCP) in the software and allows broadcast search. For example, when a user receives a research that he does not know the answer, GRP will send this research to other users.

3) RP (Routing Protocol): Through RP, users can obtain a routing path with the form of seeding messages. RP builds one or more access tables that are communicable by the way of gateways between users. The access table is used to build routers between peers. Generally, the traditional routers and DNS servers cannot build connections due to firewall and proxy servers, while RP could build connections by seeking gateways to break obstacles like firewall.

4) GCP (Group Control Protocol): Group Control Protocol provides an environment that is east for users to communicate. For example, users who are interested in a certain topic can make up a group in which one chatting service will be used to communicate. This way, the chatting messages will limit the group members. For those who want to join in this group, they can use a member's ID for authentication. If they have no such ID, then they will not join in the group and cannot have access to the chatting service of the group. The group can be seen as a virtual personal network VPN. One VPN allows the 
communication among several computers and does not allow other users on the Internet to join in. Eavesdroppers cannot understand the talk in the group as VPN is encrypted; the group can encrypt messages by limiting the accession of some users. The intra-group message transmission of GCP defines a basic protocol for users when they receive or send messages, and control the transmission of messages.

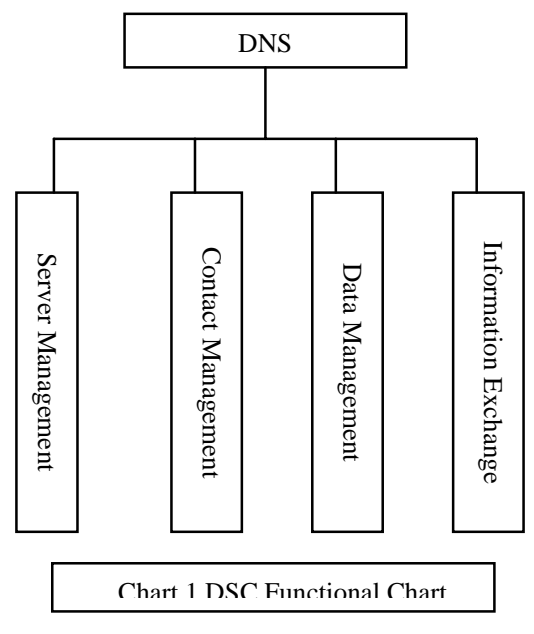

Figure 1 DSC Functional Chart

\section{ANALYSIS OF MAJOR PROBLEMS}

\section{A. Self-organization}

Self-organization means that the system could adapt automatically to the changing environment and adjust its own structure. For this system, the changing of the environment includes the accession and exit of nodes, the scale of the system, network traffic, bandwidth, breakdown and outside attack. In designing the software, we must take into account of the adaptability of the software. The typical way is to update the adjacency list of user node machine with a certain strategy, and limit the adjacency list in a certain scale to ensure the good performance of the system, and to enable the scale of the whole network not controlled by the number of local user nodes.

\section{B. Virus problem}

The sharing and routing mechanisms of the distributed server technology make it easier for network virus to hack in. users' capacity of preventing virus is different, so once one user using the software contracts the virus, the virus can spread to adjacent nodes due to the sharing and communication mechanisms. Moments later the net will be jammed or even paralyzed. Then the sharing information and confidential information are lost. What is worse, the whole net may be controlled by the virus. Therefore, we need to build a more secure system to counterattack potential network virus.

\section{CONCLUSION}

This thesis analyses the designing idea and shortages of the current popular network communication software, and presents the designing idea of a new type of network communication software and further analyses the technical completion and protocols of the software. The distributedserver-technology-based network communication software has not come into actual application, but it represents a developing trend.

\section{REFERENCES}

[1] Doreen L. Galli. Principles and Practices of a Distributed Operation System [M]. Xu Liangxian, Tang Ying, and others translated. Beijing: China Machine Press, 2003.

[2] Wu Maqun, Liu Bin. Computer Network Technology and Application [M]. Beijing: Beijng Industrial University Press, 2005.

[3] Jean Bacon Tim Harris. Operating System----Concurrent and Distributed Design [M]. Chen Xiangqun, translated. Beijing: Electronic Industry Press, 2002.

[4] Chadie Russe1. Windows NT Server 4.0 MS WORD [M]. Zi Wei, Hu Jiangping, and others, translated, Beijing: Tsinghua University Press, 2003.

[5] Li Xiaoning, Dong Hongyu, Guo Zhihao. Intemet / Intranct Technology [M]. Beijing: Higher Education Press, 2003. 